At St Mary's Hospice there was not a recognisable MDT meeting. Although in-patients were discussed weekly, it was called a "communication meeting", with little structure or outcome. On joining the organisation in 2012, I carried out a "snapshot" survey of the current MDT meeting and found that it lacked structure, outcomes, was very physically focussed, and did not include full MDT discussion. In October I wrote up my findings, stressing the positives as well as the challenges. This was shared with staff at one of these communication meetings. The response of all staff was very supportive.

I therefore devised an MDTM based on SBAR. This had been used in my previous place of work for MDT meetings but I further developed this into specific documentation, and planning paperwork (this will be shared at the conference). "Situation" covers patient information such as diagnosis, family tree, reason for referral and input; background includes relevant medical history, events leading up to referral, any other holistic issues; assessment includes what has been found / assessed since involvement, including advance care planning; recommendations include the plan of care.

The new format began trial in February and will continue until July 2013. It will then be audited: this will include staff surveys, audit of how the documentation has been utilised, and a full review. Details will be included at the conference, if this is accepted.

Early feedback shows increased attendance by the MDT, patients from all departments now being discussed, clearer documentation informing succinct relevant discussion, and clear being plans made each week for patients discussed.

\section{P80 APPLYING THE CLINICAL GOVERNANCE TOOLKIT IN THE HOSPICE SETTING; A POTENTIAL STRATEGY TO IMPROVE CARE THROUGH STAFF ENGAGEMENT IN CLINICAL GOVERNANCE}

Colin Twomey. St Wilfrid's Hospice, Eastbourne, UK

\subsection{6/bmjspcare-2013-000591.102}

Introduction Clinical governance is essential to the delivery of high quality safe and effective patient care. It provides a framework to continuously improve the quality of hospice services.

Aims To critically appraise the current clinical governance systems within our hospice and to re-design these to be efficient, transparent and encourage staff engagement.

Methods Workshops were held to examine existing governance structures and highlight areas of need and develop a solution which was both readily implementable and effective. The workshops reviewed current literature and examples of good practice surrounding clinical governance in hospices.

Results A consensus decision was reached to construct clinical governance under the 'three pillars' of patient safety, clinical effectiveness and patient experience as described by the Clinical Governance Toolbox (1). In order to enhance engagement with clinical governance three new groups were created for each 'pillar'. Each group consists of a multiprofessional membership of clinical and non-clinical staff and is Chaired by a member of the senior management team. The group members will be responsible for disseminating information and educating staff and volunteers in their day-to-day work as well as in a monthly newsletter. The Clinical Governance board, drawing together the work of the three pillars, will provide organisational oversight. The Clinical Governance Board is comprised of staff members, Trustees and an external scrutinizer.

Conclusions In adopting this clinical governance structure we believe we have developed a streamlined system that will promote best practice in all members of staff through clarity, communication, accessibility and inclusiveness. Importantly this process has also improved awareness of clinical governance throughout our team through the dynamic workshops responsible for re-designing our systems.

\section{\begin{tabular}{|l|l}
\hline P81 SIGNIFICANT EVENT ANALYSIS IN A HOSPICE SETTING \\
\hline
\end{tabular}}

Amanda Gregory, Laura Myers. St Catherine's Hospice, Crawley, UK

\subsection{6/bmispcare-2013-000591.103}

Monthly significant event analysis (SEA) sessions, which all clinical staff are invited to attend, were introduced at an independent Hospice in 2010. SEA allows opportunity for a team to come together and reflect on a clinical event that has had a significant impact for them; in either a positive or negative way. The chair of the clinical quality (now clinical audit) group introduced SEA in recognition that it forms an important part of clinical governance. Guidance was developed outlining how a significant event may be identified, how the sessions would be structured (through use of a specific proforma) and facilitated, ground rules to be followed and including background information on the role of SEA in a clinical setting. Pathways for staff support are also outlined in the guidance. Dates of SEA sessions are issued in advance. The significant event to be discussed and session facilitator are decided at the organisations' "Implementing Clinical Governance Group (ICGG)". Since it's introduction, the structure of SEA has been reviewed and adjusted to ensure it is robust from both a governance and learning perspective. This includes ensuring ground rules are set at the beginning of each session and that action points are followed up through ICGG. An aspect we are keen to further develop is the link between SEA learning outcomes and staff education. Examples of topics reflected on at SEA include management of terminal agitation, vulnerable adults, communication, boundaries, expectations, capacity and preferences. SEA is well attended and has received positive verbal, informal feedback with staff voicing they enjoy the sessions and are keen for them to continue. Recently a questionnaire has been developed and sent to all clinical staff to obtain formal feedback to support the development of future SEA sessions.

\section{P82 THE INTRODUCTION OF ELECTRONIC PATIENT RECORDS ON A HOSPICE IN-PATIENT UNIT (IPU)}

Nicola Butterfield. Birmingham St Marys Hospice, Birmingham, England

\subsection{6/bmjspcare-2013-000591.104}

Background/Context Healthcare information systems have evolved to play a major role in healthcare in modern society and the introduction of the electronic patient record aims to improve patient safety and documentation quality. SystmOne is a centralised clinical system developed by TPP (The Phoenix Partnership) and its introduction at the Hospice was due to a need to introduce an updated medical activity system that incorporated patient records. 
Aim To successfully up-skill the hospice staff and to safely and effectively introduce SystmOne onto the IPU.

Approach used The introduction of SystmOne was seen as a major change for the IPU and hence a change management model was used to support this. The main areas addressed were process mapping, to identify areas where we could work more effectively and staff training. Staff training was of paramount importance as $26 \%$ of the IPU staff had no basic IT skills. This was given to those staff before embarking upon the SystmOne training.

The ward staff were all involved in the development of new IPU documentation and the review of nursing care plans.

The change has been anchored by the alteration in ward routine, staff job descriptions and the change in hospice policies. Outcomes

- Improved communication between departments and external organisations who use SystmOne

- Reduced amount of time spent completing documentation

- Improved documentation standards

- Reduction in patients being asked the same questions on several occasions

- More than one person/department being able to access the patient notes at any one time

- Elimination of time spent searching for patient notes

- Enhanced management of out of hours phone calls thereby improving clinical safety

- Improved audit and monitoring

Application to hospice practice We were one of the first IPU's to 'go-live' with Systmone and the improvements have been wide-reaching for both patients and staff.

Nicola Butterfield

Lead Nurse, IPU

Birmingham St Mary's Hospice

\section{P83 APPLYING NHS PRODUCTIVE SERIES MODEL TO HOSPICE CARE - RELEASING TIME TO CARE}

Cathryn Goodchild, Maggie Draper. St Luke's Hospice Plymouth, Plymouth, United Kingdom

\subsection{6/bmjspcare-2013-000591.105}

Introduction Productive Ward and Productive Community are a series of modules designed to deliver sustainable service improvement, and are well established in the NHS. We decided to adopt the model, fund the education and development and apply it to:

- Hospice Inpatient Unit

- Hospice Day Care provision

- Hospice Community Palliative Care team

- Hospice Lymphodema Service

Aims To use the series across the organisation to improve efficiency and consider processes in a systematic way using a tested methodology. This will lead to reduction in time wasted, duplication, frustrations etc and the aim is that the time saved can be re-invested in direct patient care. This involves a culture shift within the organisation to adopt new ways of working and is unique within the Hospice movement. The aim is to embed new improved ways of working across the organisation and to empower staff from the "ground up".

Methods
- External training delivered

- Steering group established

- Launch in different teams and establishment of champions

- Work streams to deliver 2 modules in first year :

- Knowing How We Are Doing - public display of information e.g.: response times, no of referrals, falls, staffing levels/ sickness rates/ caseload averages/RAG rating etc.

- Well Organised Working Environment /Wardreview of referral process and pathway, evaluating stock levels and standardisation of store cupboards/ clinician's bags etc.

Evaluation The Productive Series model encourages:

- Continuous evaluation and feedback to teams.

- Ownership of changes, sustaining improvement and embedding practice

- Empowering of staff across bands and settings to deliver the organisation's vision and release time to care.

Further information to follow regarding roll out, changes adopted and impact evaluated by October 2013

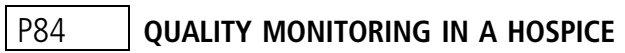

Pauline Flanagan, Michelle Roberts. Douglas Macmillan Hospice, Stoke-on-Trent, UK

10.1136/bmjspcare-2013-000591.106

Background/context Hospices are expected to quantify the quality of care and demonstrate outcome improvements ${ }^{1,2}$. There has been a change in emphasis from system and process to outcome $^{1-4}$. However, little hospice-specific guidance is available ${ }^{1-6}$.

The DMH produces quarterly quality monitoring (QM) reports and is seeing useful intelligence. Aim

- To produce hospice-specific intelligence

- To evidence that the hospice remains low risk.

Approach used QM is part of the hospice's well-established clinical governance function. Reports use monthly (\& rolling annual) data with published statistics (Office of National Statistics; National End of Life Care Intelligence Network).

Electronic patient-notes enable data collation in a way that was impossible with paper notes.

Hospice-specific outcome indicators have been developed to evidence compliance with outcome measures given in the NICE 2011 quality statements ${ }^{2}$ and the essential standards of quality and safety ${ }^{4}$.

Outcomes The hospice has robust evidence of the following:

- Number (\%) and diagnosis of patients receiving care

- Primary care aim

- Equity of access

- Impact of the 24/7 advice line

- Percentage of hospice

$\circ$ deaths of patients offered an ACP

- patients achieving their preferred place of care.

- Percentage of in-patients

$\circ$ on the LCP at the time of their death.

$\circ$ assessed for risks associated with moving and handling.

- experiencing minor/serious injury.

$\circ$ assessed for tissue viability within 6 hours of admission. 